\title{
COMPLEJIDAD Y DERECHO
}

Carlos María Cárcova

Universidad de Buenos Aires (Argentina)

\section{La noción de «complejidad»}

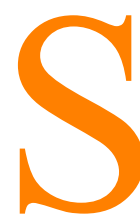

e sostiene con frecuencia que los problemas de la complejidad social conciernen a las sociedades del capitalismo maduro. Sin embargo, el fenómeno es también observable en las nuestras, aun cuando no hayan alcanzado aquel estadio de madurez, porque ellas exhiben una realidad singular, en la que conviven tradicionalismo y post modernidad; miseria y consumismo; relaciones productivas pre-capitalistas y desarrollos económico-tecnológicos de punta; analfabetismo y sofisticación intelectual, en una caleidoscópica mixtura, que se constituye, a su vez, en dato peculiar de nuestra propia complejidad.

Este concepto, el de «complejidad», ha sido particularmente estudiado en el campo de la cibernética, de la inteligencia artificial y de la teoría de sistemas en general y aplicado a los sistemas sociales, por autores con preocupaciones muy diversas ${ }^{1}$. No siempre con el mismo sentido, ni con gran precisión. De todas formas, es posible afirmar sin mayor hesitación que los fenómenos de aceleración histórica, asociados a los descubrimientos científicos y tecnológicos de las últimas décadas, han ensanchado de un modo excepcional el universo de elecciones posibles en el campo de la interacción humana. Como recuerda Raymond Aron, para llegar a Roma desde París, Napoleón empleó, prácticamente, el mismo tiempo que había empleado César; y un miembro de la burguesía francesa de la época de Luis XIV disfrutaba de los mismos recuerdos que un romano de la clase alta. Luego, las diferencias se hicieron, súbitamente, enormes².

Tenemos ante nuestra vista algunos niveles de nuestra complejidad epocal: aumento de la productividad del capital y correlativo aumento de la desocupa-

${ }^{1}$ En Zolo, Danilo. «Democracia y complejidad. Un enfoque realista». Nueva visión. Bs. As. 1994, puede consultarse una extensa bibliografía (pág. 231 y ss.)

${ }^{2}$ R. Aron. «Dix-huit leçons sur la société industrielle», París, Gallimard, 1962. En Zolo, D. op. cit. pág. 34 . 
ción, con sus deletéreos efectos sociales; densificación e incremento de la interacción social con su correlativa proliferación de códigos y multiplicidades culturales; disponibilidad del mundo a partir del conocer y monopolio del conocimiento, etc. Pero, muchos otros registros podrían, también, hablarnos de ella. Sin ir más lejos, los cambios producidos en la relación entre cultura y máquinas inteligentes. Hasta hace muy poco tiempo atrás, el mundo de las redes y los hipertextos parecían cuestión de ciencia ficción. Hoy los dispositivos multimedia, el acceso a Internet, los grupos de usuarios, los correos y diarios electrónicos, forman parte de nuestra cotidianidad. Ello comporta nuevos problemas que son, también, de naturaleza política y jurídica, vg.: el uso de la red para la divulgación de las ideas racistas de grupos nazis o filonazis o la proliferación por su intermedio de pornografía. Pero, del mismo modo, se encontrarán allí otros mensajes: Zaire o Chiapas, género y diversidad, identidad y saber, produciendo inéditas solidaridades y construyendo universos simbólicos alternativos, no por virtuales, menos eficaces.

¿Qué supondrá, para el destino próximo de nuestras sociedades, el aumento casi desmesurado de nuestras posibilidades comunicacionales, en un mundo en el que, como se ha dicho, se cruzan la sofisticación tecnocultural con la crucialidad de la miseria y la exclusión? ¿Generará el hiperdesarrollo tecnológico modificaciones significativas en el modo de aprehensión de la realidad, en el universo de valores o en las relaciones afectivas?

Por de pronto, no puede ignorarse que ese hiperdesarrollo está firmemente articulado con las estructuras de poder del mundo contemporáneo, que son las del capital financiero. Sin embargo, paradójicamente, no es la Internet lo más parecido a esa especie de desideratum democrático, que Habermas ha llamado «espacio público de comunicación». Al menos hasta hoy, se trata de un espacio descentrado, no monopolizado, multiforme y anárquico, con todo lo que esto tiene de amenaza y de posibilidad. «En algún lugar hay que poner el dato -dice, en este sentido, Aníbal Ford- de que el chico de la villa o la favela, que juega con un "video game", está recibiendo la tecnología de rezago de las investigaciones de la NASA. Ésta fue la que desarrolló los "software" de simulación para el aprendizaje, que hoy permiten u obligan a un chico semianalfabeto de un barrio pobre, a luchar como karateca en el Bronx, mientras el cartelito, irónicamente, le dice "Take the law in your hands». $O$ entrar en realidades virtuales que nunca podrán disimular sus carencias materiales».

"Sea como fuere -concluye- el aporte de las nuevas tecnologías a las formas en que se desarrollan y constituyen el conocimiento y el intercambio humanos, es una realidad insoslayable. Los monstruos ya están sueltos. Y configuran problemas inéditos. Ni la relación hombre/máquina tiene la fi- 
gura de Frankenstein o su versión Barbie (la Mujer Biónica), ni el poder, que es cada vez más duro e implacable, tiene la forma del Big Brother o del Panóptico. Es menos visible, más inteligente y no tiene centros fijos. Pero no por eso deja de actuar, de privilegiar y excluir. Y esto no es un simulacro, aunque las relaciones entre lo factual y lo simbólico, deban ser repensadas». (1995, 16 y ss.)

Desarrollo tecnológico que habilita otras formas de la comunicación humana; comunicación que acelera y transforma los flujos, produciendo impacto en las percepciones y en los procesos cognitivos; circulación del poder y del control; riesgo y posibilidad, he aquí otras dimensiones de la complejidad en la que estamos inmersos y que supone desafíos de muy diversa índole, entre otros, desafíos para las estructuras institucionales conocidas y las formas tradicionales de regulación de las relaciones sociales; desafíos, consiguientemente, para la teoría y los teóricos del derecho. Desafíos, por último, para la comprensión de tan sofisticados procesos y de las formas de legalidad que a ellos corresponden, que se toman, por razones estructurales, progresivamente más opacas.

En lo que sigue, presentaremos una visión menos impresionista y considerablemente más abstracta de la complejidad. La que ha desarrollado, desde una perspectiva sistémica y autopoiética, Niklas Luhmann. Nos parece de interés hacerlo, porque sus muy elaborados puntos de vista no han sido aún suficientemente divulgados en nuestro medio y porque, cualquiera sea el juicio de valor que ellos nos merezcan, resultan portadores de muy considerable fuerza innovativa y no menos considerable fundamentación. En el parágrafo siguiente, nos referiremos al concepto de «complejidad» en general, como categoría fundamental de una teoría de la sociedad; en el sucesivo y último, a la relación del mismo con el derecho.

\section{Complejidad sistémica}

Para poder hacer referencia, a la vez sintética e inteligible, de esta noción, será preciso comenzar por algunos de los presupuestos de la teoría luhmanniana. Como punto de partida, una constatación: el mundo es de tal manera infinito que, por serlo, resulta inabarcable, o mejor, inobservable. Tal infinitud es cortada, sin embargo, por una línea divisoria; de un lado se encuentra el sistema, del otro su entorno. Con estas nociones de «sistema» y «entorno» (o ambiente), Luhmann desplaza las de «unidad» y «partes» (componentes de esa unidad), que corresponden a una visión clásica, pero que han exhibido ya su insuficiencia, entre otras cosas, porque no dan cuenta de sus propios entornos.

Existen diversos sistemas, según el objeto de análisis que les sea propio y, aunque sólo el observador puede distinguir entre sistema y entorno, él no 
puede, en cambio, decidir acerca de qué sea sistema. Sólo el sistema, a través de sus propias operaciones, se constituye como sistema, enlazando operaciones propias con operaciones propias y, de ese modo, autorreproduciéndose. Aparece así, una primera situación paradojal, porque lo que llamamos sistema, no es más que la diferencia entre sistema y entorno, esto es, la capacidad del sistema de definir sus propios límites; lo que transforma al mismo, en parte de su propia distinción. Siendo las cosas de este modo, argumenta el autor, «... se anulan las posibilidades descriptivas de la lógica clásica bivalente y la teoría del conocimiento que la sustenta. Al igual que en la cosmología de Einstein (movimiento y aceleración), la observación del mundo con ayuda de la distinción entre sistema y entorno, depende de la ubicación del observador» (1991, p. 16).

Si el observador observa en el mundo sistemas que se reproducen a sí mismos, está obligado a considerarse como uno de ellos, pues, de lo contrario, no podría observar su propia observación. Por esta vía, la teoría de sistemas se universaliza, volviéndose una teoría del mundo que se incluye a ella misma y obligándose a concebir todo, como sistema o como entorno. Luhmann sostiene que la sociología tradicional, incluida la de Parsons, sobre la que él mismo se ha apoyado, no pudo formular desarrollos de este tipo por carecer de un aparato teórico adecuado. Sin embargo, una epistemología del tipo de la que propone, no sería vista como extraordinaria por las ciencias sociales si consultara a físicos como Heinz von Foerster, a biólogos como Humberto Maturana o a psicólogos como Jean Piaget. $\mathrm{Y}$, respecto de quienes critican la posibilidad de hacer extensivos por analogía, conceptos de otras disciplinas tales como los de «reproducción autopoiética», «clausura operativa»o «evolución», el autor les recuerda su radical relativismo sistémico pero, al mismo tiempo, les recuerda también que la noción de «proceso» fue descubierta primero en la jurisprudencia y luego aplicada a la química.

Para no fracasar, la teoría de los sistemas sociales debe transformarse de teoría de la acción en teoría de la comunicación, pero no como acción de comunicar o como transferencia de un sistema a otro. Tanto la teoría de la acción como la de la acción de comunicar, remiten a seres vivientes como conciencia, en la tradición iluminista que llega hasta nuestros días, justamente como «filosofía de la conciencia». Luhmann visualiza esta posición como teóricamente superada; corresponde a lo que denomina pensamiento «véteroeuropeo» o «paleoeuropeo», advirtiendo que la realidad social es un estado de cosas ya constituido y que la comunicación de que se trata, es la que establece cada sistema con su entorno, precisamente para resolver o atenuar la complejidad del entorno. La sociedad se describe, así, como un sistema recíproco de comunicaciones con sentido. A su vez, el entorno de 
la sociedad está constituido por todos los aspectos a los que la comunicación se refiere, lo cual implica la posibilidad de incluir otros sistemas (no sociales, sino psíquicos, físicos, biológicos, etc.). Los seres humanos resultarán, de este modo, descriptos como parte del entorno de la sociedad; no cabe, por tanto, decir que la sociedad esté «compuesta» de seres humanos (como pretendían los clásicos) ni de acciones o funciones (según la tradición que va de Weber a Parsons) ya que en realidad está, efectivamente, compuesta de comunicaciones. Con todo, no se trata de que el hombre, el ser humano, pierda relevancia en su teoría ni que se diluya como ambiente o entorno de un sistema, sino que lo que cambia en la teoría sistémica, es la situación jerárquica en que resulta ubicado, por comparación con las teorías tradicionales. Éstas, lo trataban como sujeto sin hacerse cargo de que proveían una categoría ambigua y nebulosa, exenta de referencias empíricas, a las que el hombre de carne y hueso era difícilmente reductible. La teoría de sistemas y la distinción entre sistema y entorno, permiten una respuesta a la pregunta acerca de «qué pasa con el hombre», pero ella no es la única posible ${ }^{3}$. "Sin embargo -agrega Luhmann- la situación actual de la ciencia favorece de manera considerable el intento de la teoría de sistemas, sobre todo por la gran cantidad de trabajos preliminares que pueden ser aprovechados. Comparada con este rico acerbo en conceptualizaciones, la teoría clásica de la acción da la impresión, por un lado, de simplicidad y, por otro, cuando se alía a los complicados modelos del rational choice, produce el efecto de un teatro de títeres en lugar de una teoría con pretensiones explicativas» (op. cit. p. 18).

La idea del sistema que nuestro autor desarrolla, modifica, según ya se ha insinuado, la que proviene de las elaboraciones de Bertanlanffy, quien la refería a un conjunto de elementos que mantienen determinadas relaciones entre sí y que pueden distinguirse de su ambiente. Según lo indicado, Luhmann, al percibir que la noción de sistema se contiene a sí misma, introduce el dato de la autoproducción o autopoiesis y el dato de la autorreferencialidad. Esa noción se completa con las de «observación» y «diferencia». Una observación, será siempre una operación consistente en definir un determinado esquema de diferencias. La observación sólo es posible, en otros términos, desde un determinado esquema de diferencias. Al observar, se elige uno de los aspectos que componen la diferencia y se describe cuanto se

${ }^{3} \mathrm{El}$ concepto de «sujeto» de sustrato antropológico, se convierte en sistema autorreferente basado en la conciencia y en el lenguaje, lo que plantea importantes novedades teóricas. (Cfr. Ignacio Izuzquiza, "La sociedad sin hombres. N. Luhmann o la teoría como escándalo», Anthropos, Barcelona, 1990. Para una muy interesante conceptualización de la crítica del autor a las concepciones «humanistas» puede verse «Individuo, individualidad, individualismo». en Zona Abierta $\mathrm{n}^{\circ}$ 70/71, año 1995 , pag. 53 y ss. 
ve desde ese aspecto. Por ejemplo, en el «Prefacio a la Crítica de la Economía Política», Marx decía que la «población» no podía verse con los ojos de la cara; que si uno quería «ver» la población, debía distinguir las distintas clases sociales de las que estaba compuesta, lo que conduciría a nuevas diferenciaciones como las de capital, trabajo, salario, plusvalor, etc. A partir de la articulación congruente de esas distinciones, sería entonces posible volver a la población y percibirla como «totalidad concreta».

La observación es una actividad fundamental de los sistemas autorreferentes, mediante la cual ellos se observan a sí mismos y observan su entorno, para intervenir con procesos que establecen selecciones de las diferencias. Tales sistemas autorreferentes pueden ser de tres tipos: sistemas vivos, sistemas psíquicos (o personales) y sistemas sociales. Cada uno se caracteriza por su propia operación autopoiética. Así, la vida y las operaciones vitales son propias de los primeros; la conciencia, es propia de los segundos; y la comunicación, es el rasgo característico de los sistemas sociales. La sociedad, objeto del estudio luhmanniano, es concebida, entonces, como un sistema autorreferente y autopoiético que se compone de comunicaciones. Su evolución se produce temporalmente, a través de un proceso constante de diferenciaciones que van creando subsistemas, tales como el derecho, la economía, la política, la religión, la educación, etc. Las relaciones entre ellos se regulan de forma autónoma (aunque no de forma independiente), por cada subsistema. Por ello, el sistema jurídico no es independiente de otros subsistemas pero tiene una gran capacidad de reformular en sus propios términos conflictos que aparecen en otros subsistemas. Por ejemplo, retraduce conflictos de tipo económico en términos legales, y los regula ulteriormente según un criterio de naturaleza jurídica. A su vez, el subsistema económico reconstruye internamente acciones legales en sus propios términos. Este fenómeno marca una nueva situación paradojal: la apertura de cada subsistema, su percepción del entorno, se funda en su clausura, es decir, en la autonomía de sus operaciones internas. Ellos se constituyen en la medida de su clausura, es decir, de su diferenciación del entorno, al que, sin embargo, se abren, mediante prestaciones selectivas dirigidas a resolver la complejidad creciente del entorno. En esta tarea, los subsistemas especializan el ámbito de sus comunicaciones y de sus selecciones de un modo tal, que cada uno de ellos resuelve una parte definida de la complejidad del ambiente. Esa resolución, es imprescindible a los efectos de suprimir o paliar un riesgo, que es propio de la evolución temporal de la sociedad, que es el riesgo de la entropía. Luhmann, explica esa evolución como un tránsito de sociedades elementales a sociedades altamente diferenciadas, que especializan sus ámbitos de selección y son enormemente complejas, a la manera de nuestras sociedades actuales. Las operaciones autorreferentes de los sis- 
temas no pueden sino apoyarse sobre anteriores operaciones de la misma naturaleza. Por ejemplo, las decisiones políticas, en tanto elementos del sistema político, sólo son posibles sobre la base de decisiones políticas anteriores y son consideradas tales, en relación con una red de decisiones del mismo tipo, que se diferencian de las religiosas, económicas, etc. De este modo, el sistema social reduce complejidad, pero en forma contingente, al seleccionar sólo algunas de una multiplicidad de alternativas «funcionalmente equivalentes». Lo que, por otro lado, explica que sociedades semejantes se den para sí soluciones distintas y tengan, por ello, evoluciones notoriamente diferenciadas. La idea de autonomía de cada subsistema social implica, además, que no existe entre ellos relaciones «jerárquicas», no existe centralidad de uno respecto de los otros. Todos son igualmente imprescindibles, porque realizan alguna actividad que resulta crucial para el conjunto, pero sin que se establezcan supremacías. Esa actividad está orientada, tal como se ha reiterado ya, a reducir complejidad.

La complejidad consistirá, entonces, en la forma en que se relacionan los elementos de un sistema. Cuando la cantidad de los mismos aumenta, ya no es posible relacionar cada uno de los elementos, en el mismo momento, con cada uno de los otros, generando así una situación de complejidad. Con su prosa habitualmente críptica, Luhmann sostiene: "Complejidad en el sentido mencionado, significa coacción de la selección. Coacción de la selección significa contingencia, y contingencia significa riesgo. Cualquier estado complejo de cosas se basa en una selección de las relaciones entre los elementos, los cuales, a la vez, son utilizados para constituirse y conservarse. La selección sitúa y cualifica los elementos, aunque para éstos fueran posibles otras formas de relación. Designamos este «ser posible también de otro modo» mediante un término cargado de tradición, que es el de contingencia. La contingencia advierte sobre la posibilidad de error aun en la mejor posibilidad relacional de los elementos» (1991, 47) [hemos introducido correcciones menores en la construcción sintáctica del párrafo, según resulta de la traducción correspondiente a la edición citada, porque, en nuestra opinión, ayudan a la comprensión del mismo $]^{4}$.

${ }^{4}$ Como el propio Luhmann admite en el prefacio a la traducción española, su texto es difícil. Sin embargo, para quienes no manejamos el idioma del autor, sobrevive la duda respecto de qué parte de responsabilidad cabe atribuirle a él mismo y qué parte concierne a sus traductores. Un buen ejemplo de ello puede encontrarse en la comparación de la traducción que realizan del primer capítulo de «Sistemas Sociales» Santiago López Petit y Dorothee Schmitz, para la monografía dedicada a Luhmann, con prólogo de I. Izuzquiza, que Paidos editara en 1990, con la traducción de la obra completa, que realizaran S. Pappe y B. Erker, bajo coordinación de Javier Torres Nafarrate, para la edición de Universidad Iberoamericana-Alianza Edit., México, 1991. De esa comparación, surgen insólitas diferencias entre ambas versiones. 
Una definición más «amigable» del concepto de complejidad, es propuesta en el glosario que el propio Luhmann elaboró para su obra «Ökologische Kommunikation» y que fuera traducido por Verónica Muñoz Dardé $(1995,17)$, en los siguientes términos: «Un hecho es complejo si consiste en tantos elementos que éstos pueden estar en relación recíproca, sólo en forma selectiva. Tanto en el plano operativo como para la selección, la complejidad presupone siempre un proceso de reducción que fija un modelo de selección de las relaciones, excluyendo temporalmente otras posibilidades de conexión de elementos como meras posibilidades potenciales».

La complejidad ayuda a percibir la diferencia entre sistema y entorno, porque para cualquier sistema el entorno resulta más complejo. A los sistemas les falta la «variabilidad requerida» necesaria para poder acompañar cualquier modificación del entorno. En otros términos, no hay coincidencia entre sistema y entorno.

Ahora bien, como dice $\mathrm{D}$. Zolo, cuanto más amplio es el número de las posibles elecciones y más elevado el de las variables que los agentes deben tener en cuenta, en sus intentos de resolver problemas de conocimiento, adaptación y organización, más compleja se vuelve su situación en el medio (1994, p. 17). A su vez, el medio ambiente o entorno aumenta en complejidad cuanto más interdependientes se tornan sus variables. Ello demanda, entonces, una mayor información para disponer y controlar ese entorno. A este esquema de complejidad en aumento, se suman otros dos elementos, coadyuvantes al mismo fin: por una parte, la denominada inestabilidad o turbulencia del ambiente, que introduce en el campo de la ciencia las cuestiones de la imprevisibilidad, el caos, la catástrofe, etc. ${ }^{5}$; y por la otra, el estado de circularidad cognitiva alcanzado por los agentes al registrar la complejidad del medio. Ello les permite advertir que no pueden captarlo objetivamente, sin incluir la distorsión que su propia presencia cognitiva produce. Se genera así, lo que Zolo ha llamado complejidad epistemológica y para la que ha propuesto una «epistemología reflexiva» ${ }^{6}$.

En conclusión, en el análisis sistémico, el mundo ofrece al observador una cantidad prácticamente ilimitada de posibilidades de experiencia y de acción, a la cual corresponde, en cambio, una capacidad muy reducida de percibir, elaborar información y actuar. La «complejidad» no es más que

${ }^{5}$ v. en ese sentido, las nociones de «orden a través del ruido» de H. Atlan («Entre el cristal y el humo») o de «estructuras disipativas» de I. Prigogine («La Nueva Alianza»). También v. George Balandier, «El Desorden-La teoría del caos y las ciencias sociales», Gedisa, Barcelona, 1989.

${ }^{6}$ No se trata, en realidad, de una novedad epistemológica. Entre otros autores el tema ha sido tratado por Lucien Goldmann en «Las Ciencias Humanas y la Filosofía», Ed. Nva. Visión, BsAs., 1972 , p. 21 y ss. 
«exceso de las posibilidades del mundo». Es en tal sentido, como se indicó más arriba, que la complejidad implica necesidad de selección. A través de la selección se alcanza la estabilidad del sistema. Los sistemas reducen la complejidad del ambiente, seleccionando las opciones necesarias para sus fines, pero al hacerlo, transforman la complejidad externa en complejidad interna, produciendo una nueva situación de naturaleza paradojal. El ciclo así descrito, es un círculo virtuoso en el sentido de que implica un aumento permanente de la complejidad de los sistemas (Cfr. N. Luhmann, 1991). El círculo vicioso es, en cambio, el que no aumenta la complejidad. Deberá tenerse aquí en cuenta que, para nuestro autor, sólo el adecuado nivel de complejidad de un sistema social opera como garantía de la justicia. La justicia es una consecuencia del adecuado nivel de complejidad de un determinado sistema social ${ }^{7}$.

Permítasenos intentar, en el desarrollo del próximo parágrafo, hacer inteligible esta final observación.

\section{Derecho y complejidad}

Luhmann es notoriamente reacio a descender del nivel de alta abstracción de sus formulaciones. Sin embargo, en su «Rechtssoziologie» (1983) sugiere como ejemplo del aumento creciente de la complejidad de un sistema, aunque no lo desarrolle, el implicado en la evolución y progresiva diferenciación del derecho occidental.

Como es sabido, los romanos abarcaban jurídicamente el conjunto de las relaciones sociales de su época, a través de la distinción entre el jus quiritario (derecho de la ciudad) y el jus gentium (derecho de gentes/extranjeros); al proceso histórico de diferenciación funcional, fue correspondiendo también un proceso de diferenciación y progresiva automatización del derecho: el derecho civil, del cual se desprenden el derecho mercantil y más tarde, el de la navegación, el laboral, aeronáutico, minero, fiscal, etc. Cada campo de especialización, permite abarcar nuevas relaciones y, con ello, seleccionar opciones y reducir la complejidad del ambiente, a costa de aumentar la propia complejidad de ese campo, al incrementar su información interna, generar estándares interpretativos ad-hoc, etc.

Piénsese en los desafíos que a la estructura actual de los sistemas de decisión jurídica, plantean las innovaciones científicas y tecnológicas con la

${ }^{7}$ En Edgar Morin puede encontrarse una idea bastante próxima. El abandono del círculo vicioso y su reemplazo por la circularidad virtuosa, es la condición para la generación de un pensamiento complejo. La circularidad abre la posibilidad de un método que permite la interacción de los términos que remiten los unos a los otros productivamente, a través de procesos y cambios de un conocimiento complejo que comporta su propia reflexividad. v. «La Methode», vol. I, Seuil, París, 1977, p. 19. 
aparición de cuestiones como los delitos ambientales, los ilícitos informáticos, la regulación de la telemática, la robótica, la fecundación «in vitro», la bio-tecnología en general, etc. Cada uno de estos campos, de estos elementos del subsistema jurídico se especializa, en un proceso que es constante y que permite controlar la diferenciación producida en el entorno, es decir en el universo de las relaciones humanas, también ellas, constantemente diversificadas.

Para Luhmann, ese control se hace efectivo a través del derecho moderno positivado, mediante la «generalización congruente de expectativas». Tanto más eficaz será el subsistema jurídico, cuanto mayor sea el grado de su diferenciación interna. Sin embargo, tal proceso de diferenciación que supone un correlativo aumento de la complejidad, debe tener un límite que impida su autodestrucción, como consecuencia de la sobrecarga de prestaciones contradictorias requeridas por los otros subsistemas. Ese límite es el que nuestro autor vincula con la idea de «justicia». Ella no remite ya a un criterio ético, sino a un criterio de «consistencia» del sistema en relación con las decisiones que provee. El sistema no provee decisiones consistentes, cuando violenta el principio de igualdad, es decir, cuando por cualquier operación propia, precluye la posibilidad ulterior de decidir, del mismo modo como ya ha decidido antes, un caso de naturaleza similar.

Ciertamente, la cuestión que se plantea pasa a ser la de definir cuándo un caso es igual a otro. Es en este aspecto, en el que la ciencia del derecho, la Dogmática Jurídica, cumple un papel sobresaliente, al elaborar conceptos y clasificaciones que facilitan el trabajo del operador jurídico, investido de la función de decidir. Así considerada, la Dogmática aparece como lo opuesto de la Justicia. Mientras ésta expresa la unidad del sistema, aquella refleja su complejidad. Dice Pilar Giménez Alcover: «El sistema jurídico ha de poder conjugar un alto grado de complejidad interna para responder a las demandas de otros subsistemas sin colapsarse, es decir sin quebrar su propia coherencia interna o la integración de sus decisiones. La expresión "justicia" reflejaría la unidad del sistema pese a su complejidad, esto es, pese al número de decisiones, a su diversidad, a la interdependencia interna de las mismas, a su grado de generalización y a la velocidad de cambios a que están sometidas» (op. cit. p. 285).

Por todo esto, es que el concepto de complejidad aparece estrechamente vinculado al concepto de derecho. André J. Arnaud sostiene que, siendo la toma de decisión el centro de gravedad tanto del derecho como de la complejidad, una teoría de la decisión jurídica debe abordarse desde la teoría de la complejidad. Las visiones tradicionales acerca de la decisión jurídica estuvieron vinculadas, según este autor, al criterio definido como one best way: la mejor elección de entre todas las posibles. Se trata, dice, de un sis- 
tema analítico de decisión, basado en una aproximación epistemológica de tipo positivista. Pero hoy se tornan predominantes los procesos de decisión compleja, fundados en una epistemología constructivista que reivindica criterios de unidad y apertura sistémica, junto con recursividad transformacional y perspectiva teleológica (1996, p. 320). Al incorporar la perspectiva teleológica, Arnaud se separa de Luhmann de manera bastante clara. Su pensamiento en torno a la complejidad recurre a otros presupuestos, considerablemente menos abstractos. Pero, como Luhmann y otros pensadores contemporáneos, pone énfasis en la idea de autorreferencialidad, recursividad o autología del sistema jurídico ${ }^{8}$.

También es éste el caso de Günter Teubner, frecuentemente considerado en el campo específico de la teoría jurídico-política como discípulo de Luhmann. El derecho, sostiene, es indeterminado porque no es susceptible de control externo. «La ley no está determinada ni por autoridades exteriores a ella, ni por la autoridad de los textos, ni por el poder de las palabras, ni por la ley natural o la revelación divina; la ley está determinada de manera autorreferente, descansa sobre su propia realidad positiva. La ley debe su validez a esta autorreferencialidad: la aplicación de operaciones legales a los resultados de las operaciones legales. Por tanto, la validez del derecho no puede ser importada desde fuera sino solamente producida desde dentro del derecho» (1995, p. 160). En el divulgado ensayo que citamos, Teubner critica las posiciones de autores que pertenecen a la corriente de los critical legal studies, por entender que frente al derecho no son suficientemente críticos, toda vez que constatan los problemas de autorreferencialidad y paradojalidad del derecho, pretendiendo su neutralización mediante una deconstrucción que caracteriza como «ilustrada». La teoría autopoiética, en cambio, afirma que la circularidad es un problema de la práctica legal más que un problema de pensamiento legal: la realidad social del derecho -argumenta- está constituida por una serie de relaciones circulares. Los elementos del sistema legal (acciones, normas, procesos, identidad, realidad legal) están conectados de una multitud de maneras. Autorreferencia, paradojas e indeterminaciones son problemas reales de los sistemas sociales, no errores en la reconstrucción mental de esta realidad social (v. op. cit. p. 169).

La autorreferencia deja, en esta tesitura, de ser considerada como un modo de pensar prohibido o que conduciría a resultados falsos o infundados o, de cualquier manera incorrectos, como sostendría una perspectiva

${ }^{8}$ v. François Ost «Entre ordre et désordre» le jeu du droit. Discussion du paradigme autopoietique appliqué au droit» en «Archives du Philosophie du Droit», no 31, 1986, p. 133 y ss. También F. Ost y Michel Van Der Kerchove «Le systeme juridique entre ordre et désordre», PUF, París, 1988. Helmut Willke «Systemtheorie», UTB Fischer, Stuttgart, 1982. 
analítica. Ella pasa a ser, en cambio, la clave de un salto cualitativo, no sólo de la teoría legal, sino del pensamiento social en su conjunto. Citando a Danilo Zolo (1995, p. 201) Teubner sostiene que la base teórica de tal concepción está en la generalización de los siguientes fenómenos «circulares»: i) la autorreferencia lingüística de los procesos cognoscitivos (W. V. O. Quine y Neurath); ii) la circularidad lógica de los constructos de la axiomatización formal de las matemáticas (Gödel) y más en general de las paradojas de la recursividad lógico-lingüística (Russell y Tarski); iii) las teorías del orden a través de fluctuaciones y de las estructuras disipativas en la física de los procesos irreversibles (Prigogine); iv) la reflexividad de los mecanismos de autorregulación homeostática o autocatalítica en biología molecular y en neurofisiología (Bertanlanffy, von Foerster); v) la retroactividad en cibernética de primer y segundo grado (Ashby, Foerster); vi) los procesos de morfogénesis y autoorganización de los grupos sociales (Hayek); vii) la autoconsciencia psíquica de los sujetos individuales, en los primates antropomorfos y en el hombre (Maturana, Luhmann).

Como el propio Teubner señala, así abordada la autorreferencia, terminará proponiendo un salto epistemológicamente muy arriesgado que es el que Luhmann presenta del siguiente modo: «la realidad tiene una estructura circular, independientemente de su cognición» (1991, 435 y ss).

Otro de los autores que ha dedicado páginas muy interesantes al tema de la circularidad en el derecho, es Jesús Ignacio Martínez García (1992), quien muestra ciertos paralelismos entre la teoría autorreferencial y la semiótica de Greimas, en tanto horizontes de sentido susceptibles de utilizarse para percibir lo jurídico de un modo constructivista y registrar la capacidad que posee, para generar su propia realidad.

«Abordar semióticamente el problema del sentido -dice este autor- prescindiendo de un referente externo al propio lenguaje, y observar sistémicamente el dinamismo de circuitos autorreferenciales es una invitación a revisar las concepciones demasiado simplistas de las relaciones del derecho consigo mismo y con el resto de la realidad social. Los procedimientos operativos del derecho no son lineales y no pueden describirse adecuadamente recurriendo a modelos deductivos o a esquemas de causa-efecto. La realidad social no constituye sencillamente la referencia inmediata del derecho. Es cierto que el derecho cuenta con la realidad, la regula, la modifica y a su vez, se ve modificado por ella. Pero se trata de dar cuenta de un modo técnico y a la altura de la moderna epistemología del sentido de estos fenómenos. Como resultado de tales aproximaciones emerge el protagonismo del derecho, considerado ya sea como universo semiótico o como sistema autopoiético» (op. cit. p. 62). 
Por cierto, las concepciones recursivas han sido rechazadas por las tradiciones positivistas y analíticas que ven en ellas perturbaciones del orden lógico, concebido lineal y jerárquicamente, a la manera del sistema kelseniano, que instrumenta una cuidada distinción de niveles normativos y de correlativas delegaciones de poder. Con todo, el propio Kelsen percibe con agudeza el fenómeno de que el derecho regula su propia creación y que en ocasiones produce autoatribuciones de significado, como cuando los contratantes establecen los antecedentes del negocio jurídico que crean o cuando el testador describe sus disposiciones de última voluntad. Sólo puede considerarse fundamento de validez de una norma, otra norma del sistema. En Hart, es a través de la Regla de Reconocimiento, como resulta posible identificar la validez de las reglas del sistema. Alf Ross, en cambio, ha rechazado drásticamente la autorreferencialidad normativa por considerarla lógicamente inadmisible $(1969, \text { p. } 49 \text { y ss. })^{9}$.

Sin dudas, la recursividad ha producido «malestar en la teoría» pero, de hecho, la teoría se ocupa de ella desde hace mucho tiempo. Por ejemplo, cuando habla sobre lenguajes, cuando produce medios de producción, cuando investiga acerca de los procesos de investigación, cuando decide sobre premisas decisorias o cuando dicta normas que regulan la aplicación de otras normas. Como argumenta Martínez García en su paráfrasis de Luhmann, «...La circularidad no es solo una propuesta teórica entre otras muchas, sino una realidad: la consecuencia de la evolución de la sociedad. Aparece aqui como una de las más importantes conquistas del derecho moderno. Implica que el derecho se ha emancipado de otro tipo de racionalidades y cuenta con enfoques y respuestas propias frente a sus problemas. En una sociedad altamente diferenciada y con ámbitos de comunicación especializados el derecho necesita y logra en buena medida ser capaz de controlar por sí mismo, sin ayuda externa, sus propias reglas de funcionamiento». (op. cit. p. 85).

El derecho se caracteriza, de este modo, no por sus contenidos sino por sus formas, sus procedimientos y relaciones. Su dinamismo, en sentido kelseniano, supone la contingencia y con ella, la posibilidad de producir todos los cambios requeridos por el ambiente. Y su circularidad, su autología es el canon de su validez. Como dice Luhmann, validez es circularidad.

Y bien, autorreferencia, autopoiesis, diferenciación, indeterminación, complejidad, son nociones que abren la posibilidad de nuevas investigacio-

${ }^{9}$ El tema ha sido considerado por Ricardo Guibourg en «La autorreferencia normativa y la continuidad constitucional», incluido en «El lenguaje del derecho: Homenaje a Genaro R. Carrió»», Abeledo-Perrot, BsAs., 1983. También en Carlos S. Niro «La validez del derecho», Astrea, BsAs, 1985. 
nes acerca de lo social y lo jurídico, nuevas conceptualizaciones, transformaciones paradigmáticas, etc. Como se ha visto, aunque Niklas Luhmann sea una referencia insoslayable, no hay una versión canónica de las concepciones sistémicas. Danilo Zolo no ha vacilado en atacar con dureza el componente autopoiético de la teoría de Luhmann y en aconsejar su abandono, y este último, afecto a la polémica, no ha tardado en refutar sus críticas. Por otra parte, muchos autores, como es el caso de Eligio Resta, han tomado elementos de la teoría, para desarrollar un pensamiento original y novedoso, tal como resulta ilustrado por sus obras más recientes ${ }^{10}$. Cuál sea el aporte definitivo de los nuevos enfoques es aún material controversial, pero ellos no pueden ser ignorados. Han ocupado un lugar estratégico en el conocimiento del mundo que, al mismo tiempo, aparece como constitutivo del mundo. Y han enfatizado, como ninguna otra concepción precedente, el tema crucial de nuestro tiempo: la complejidad. Al hacerlo, han permitido advertir, en lo que al derecho concierne, el aumento incesante de opacidad, que acompaña al incremento también incesante de complejidad, como consecuencia de la mayor diferenciación del subsistema jurídico, de la variabilidad en aumento de sus posibilidades internas, de la especialización constante de sus prestaciones y de la singularización permanente de sus comunicaciones.

Para concluir, quizá convenga traer a cuento de una manera menos árida la presencia permanente de la complejidad, evocando el diálogo imaginado por Italo Calvino en «Las Ciudades Invisibles»:

Marco Polo, describe un puente, piedra por piedra.

- ¿Pero, cuál es la piedra que sostiene el puente? -pregunta Kublai Jan.

-El puente no está sostenido por esta piedra o por aquella -responde Marco-, sino por la línea del arco que ellas forman.

Kublai permanece silencioso, reflexionando. Después añade:

-¿Por qué me hablas de las piedras? lo único que me importa es el arco.

Polo responde: -Sin piedras no hay arcos.

${ }^{10}$ Cfr. «Poteri e diritti», Giappicheli Editore, Torino, 1996 (346 pag.) y «Le stelle e le masserizie. Paradigmi dell'osservatore», Laterza, Roma, 1997 (235 pag.). 